\title{
Role of Bedside Ultrasonography in Assessment of Diaphragm Function as a Predictor of Success of Weaning in Mechanically Ventilated Patients
}

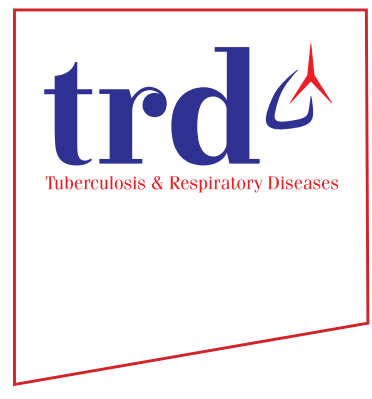

\author{
Mostafa Ibrahim Elshazly, M.D. ${ }^{\circledR}$, Khaled Mahmoud Kamel, M.D., Reem Ibrahim Elkorashy, M.D., \\ Mohamed Said Ismail, M.D., Jumana Hesham Ismail, M.Sc. and Hebatallah Hany Assal, M.D. (1) \\ Department of Chest Medicine, Faculty of Medicine, Cairo University, Cairo, Egypt
}

Background: Weaning failure is common in mechanically ventilated patients, and if ultrasound can predict weaning outcome remains controversial. The purpose of this study was to evaluate the diaphragmatic function (thickness and excursion) measured by ultrasound as a predictor of the extubation outcome.

Methods: We included 62 mechanically ventilated patients from the chest intensive care unit in this study. Sixty-two patients who successfully passed the spontaneous breathing trial (SBT) were enrolled. The transthoracic ultrasound of the diaphragm was performed during an SBT to the assess diaphragmatic function (excursion and thickness), and they were classified into the successful extubation group and the failed extubation group.

Results: There was a statistically significant increase in the successful extubation group in the diaphragmatic excursion and thickness fraction $(\mathrm{p}<0.001)$, a statistically significant negative correlation between the diaphragmatic function and the duration of the mechanical ventilation, and a statistically significant negative correlation between the diaphragmatic excursion and the Acute Physiology and Chronic Health Evaluation II. The diaphragmatic excursion cutoff value predictive of weaning was $1.25 \mathrm{~cm}$, with a specificity of $82.1 \%$ and a sensitivity of $97.1 \%$ respectively, and the diaphragmatic thickness cut-off value predictive of weaning was $21.5 \%$, with a specificity of $60.7 \%$ and a sensitivity of $91.2 \%$, respectively.

Conclusion: The diaphragmatic ultrasonography was found to be a promising tool for predicting the extubation outcome for mechanically ventilated patients.

Keywords: Weaning; Transthoracic Ultrasound; Diaphragmatic Excursion; Diaphragmatic Thickness Fraction; Successful Extubation; Failed Extubation

Address for correspondence: Hebatallah Hany Assal, M.D.

Department of Chest Medicine, Faculty of Medicine, Cairo University, Al Kasr Al Aini, Old Cairo, Cairo 11956, Egypt

Phone: 20-1223939861, Fax: 20-38642085

E-mail: Hebatallah.Assal@kasralainy.edu.eg

Received: May. 1, 2020

Revised: Jul. 13, 2020

Accepted: Aug. 10, 2020

Published online: Aug. 28, 2020

(c) It is identical to the Creative Commons Attribution Non-Commercial License (http://creativecommons.org/licenses/by-nc/4.0/).

\section{Introduction}

Weaning failure is associated with prolonged mechanical ventilation and intensive care unit (ICU) stay as well as increased hospital mortality ranging between $40 \%$ and $50 \%^{1,2}$. Delay in weaning from ventilator increases the inherent risks of mechanical ventilation, such as barotrauma, ventilatorassociated pneumonia, and ventilator-induced diaphragmatic atrophy. On the other hand, premature discontinuation of mechanical ventilation is equally as harmful and associated with mortality rates of about $42 \%^{3}$. Therefore, discontinuing mechanical ventilation in a timely and safe manner is of major importance, and strategies that assist in discontinuation 
should be thoroughly evaluated ${ }^{4}$.

Many weaning parameters have been used to predict weaning failure, including rapid shallow breathing index (RSBI) which is the ratio of respiratory frequency to tidal volume, minute ventilation, maximum inspiratory pressure, tracheal airway occlusion pressure of 0.1 second (P 0.1), dynamic compliance (Cdyn), respiratory rate (RR), oxygenation, maximum inspiratory pressure, and dynamic compliance, respiratory rate, oxygenation, maximum inspiratory pressure (CROP) index, but none has shown great prognostic accuracy ${ }^{5}$. Moreover, these parameters do not directly reflect diaphragmatic function, which is measured by diaphragmatic thickness fraction (DTF) and diaphragmatic excursion (DE) ${ }^{6}$.

The diaphragm is the principal respiratory muscle in humans and is subjected to various adverse factors in critical patients, such as hypoxia, sepsis, electrolyte imbalance, and neuromuscular blocking agents in addition to mechanical ventilation, which causes ventilator-induced diaphragmatic dysfunction by decreasing the force-generating capacity of the diaphragm ${ }^{7}$. Several diaphragm function evaluation techniques have been previously investigated, such as fluorosco$\mathrm{py}^{8}$, phrenic nerve stimulation ${ }^{9}$, dynamic magnetic resonance imaging $^{10}$, and trans-diaphragmatic pressure measurement ${ }^{11}$. However, the cumbersome nature of these techniques, ionizing radiation exposure, invasiveness, and need for patient transportation has limited their widespread use ${ }^{12}$. In contrast to those methods, ultrasound is ionizing radiation-free, noninvasive, inexpensive, and available at bedside, so it is a useful technique for assessment of the dynamic and structural function of the diaphragm. Several ultrasound techniques, such as B-mode and M-mode, have been used to assess diaphragm sonographic predictors: $\mathrm{DE}$, which measures the distance that the diaphragm is able to move during the respiratory cycle, and DTF, which is the ratio between the difference in thickness from inspiration and expiration divided by the thickness on expiration ${ }^{13}$. DTF and DE during respiration reflect diaphragm function. However, the results are controversial, and some studies have reported that DE and DTF had high sensitivity and specificity in predicting weaning outcome ${ }^{14}$.

The aim of the study was to determine if measurement of diaphragmatic function by ultrasound may be used to predict successful extubation and to determine cut-off values for predicting success or failure of extubation. We also evaluated the performance of these ultrasound parameters in comparison with conventional parameters routinely used to predict successful weaning from mechanical ventilation.

\section{Materials and Methods}

This study was conducted in 62 patients who were mechanically ventilated in the respiratory ICU, Department of Chest Medicine, Kasr Al-Aini Hospital, Cairo University between September 2017 and January 2019. The study was approved by the institutional review board at Kasr Al-Aini Hospital, Cairo University (IRB \#N-93-2018) and informed consent was obtained from the relatives of all enrolled patients. Patients who were mechanically ventilated invasively for more than 48 hours and fulfilled the criteria of weaning (hemodynamic stability, RR $<35$ breaths per minute, and hemoglobin level $>7$ $\mathrm{g} / \mathrm{dL})^{5}$ and succeeded the spontaneous breathing trial (SBT) were included in the study. Patients with diaphragmatic paralysis proved by transthoracic ultrasound and patients with neuromuscular diseases, such as muscular dystrophy, poliomyelitis, and myasthenia gravis, were excluded from the study.

Demographics, clinical information, and Acute Physiology
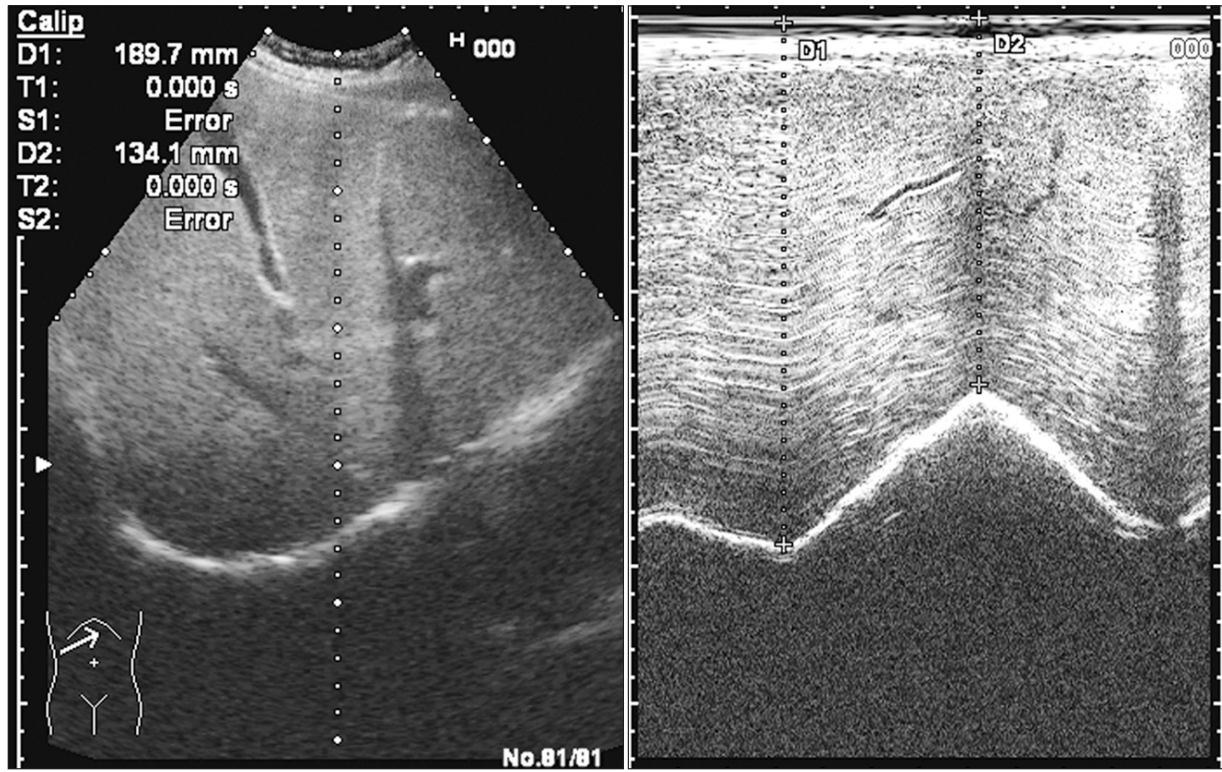

Figure 1. Upward flexion of the tracing in M-mode corresponding to the diaphragmatic movement. 
and Chronic Health Evaluation II (APACHE II) score were recorded on admission.

The patients were ventilated using volume-controlled ventilation (tidal volume $[\mathrm{TV}]=6 \mathrm{~mL} / \mathrm{kg}$ ). Assessment of lung mechanics while the patients were on mechanical ventilation was performed by recording the tidal volume, RR, RSBI calculated by dividing the RR on tidal volume, static compliance (Cstat) calculated by dividing the TV on plateau pressure minus positive end expiratory pressure, and Cdyn calculated by dividing the TV on peak pressure minus positive end expiratory pressure.

Diaphragms were visualized by ultrasound during an SBT.

All transthoracic ultrasound (Logiq P3, GE Healthcare, Chicago, IL, USA) evaluations were performed with the patients in a semi-sitting position exposing the chest and abdomen to look for the following:

\section{Diaphragmatic excursion}

A curvilinear transducer using the low-frequency of $3.5 \mathrm{MHz}$ was placed on the right sub-costal angle between the midclavicular line and anterior axillary line during quiet breathing and directed medially, cranially, and dorsally to achieve the best view of the right hemidiaphragm by using the liver as a window. The movements of the right hemidiaphragms were recorded by M-mode sonography in real time. After ensuring quiet regular breathing by the patient, we froze the sonogram. During M-mode imaging, the normally functioning diaphragm is represented as an echogenic line that moves freely during inspiration and expiration. Inspiration is identified on the sonographic tracing as upward flexion (during inspiration, there is downward movement of the diaphragm: i.e., toward the probe, leading to upward flexion of the tracing) (Figures 1,2). Expiration is identified as downward flexion (during expiration, there is upward movement of the diaphragm: i.e., away from the probe, leading to downward flexion of the tracing toward the baseline; the baseline represents the expiratory pause that follows expiration).

On the M-mode images, the distances between the probe and copula during inspiration and resting expiration were measured, and the difference between the two distances equaled the DE range (Figures 1,2).

\section{Diaphragmatic thickness}

High-frequency linear probe with a frequency of 7-12 MHz was used to measure the diaphragm thickness (DT) at the zone of apposition. The patient was placed in a semisitting position. The probe was placed at the anterior axillary line in the longitudinal plane between the seventh and ninth intercostal spaces to measure the DT. The liver window was used to visualize the diaphragm. The right DT was measured as there was no significant difference in thickness between the right and left sides. On B-mode imaging, the diaphragm appears as a thick echogenic linear structure between highly reflective pleural and peritoneal membranes. Thickness was measured by placing calipers on reflective lines at the end of inspiration and expiration (Figure 3). Thickness at end inspiration (Insp DT) as well as thickness at end expiration (Exp DT) were measured. The DTF was calculated according to the following formula:

DTF $=[(\operatorname{Insp}$ DT-Exp DT $) / \operatorname{Exp}$ DT $] \times 100$

Patients were extubated when able to tolerate the SBT for 2 hours without exhibiting clinical signs of respiratory fatigue
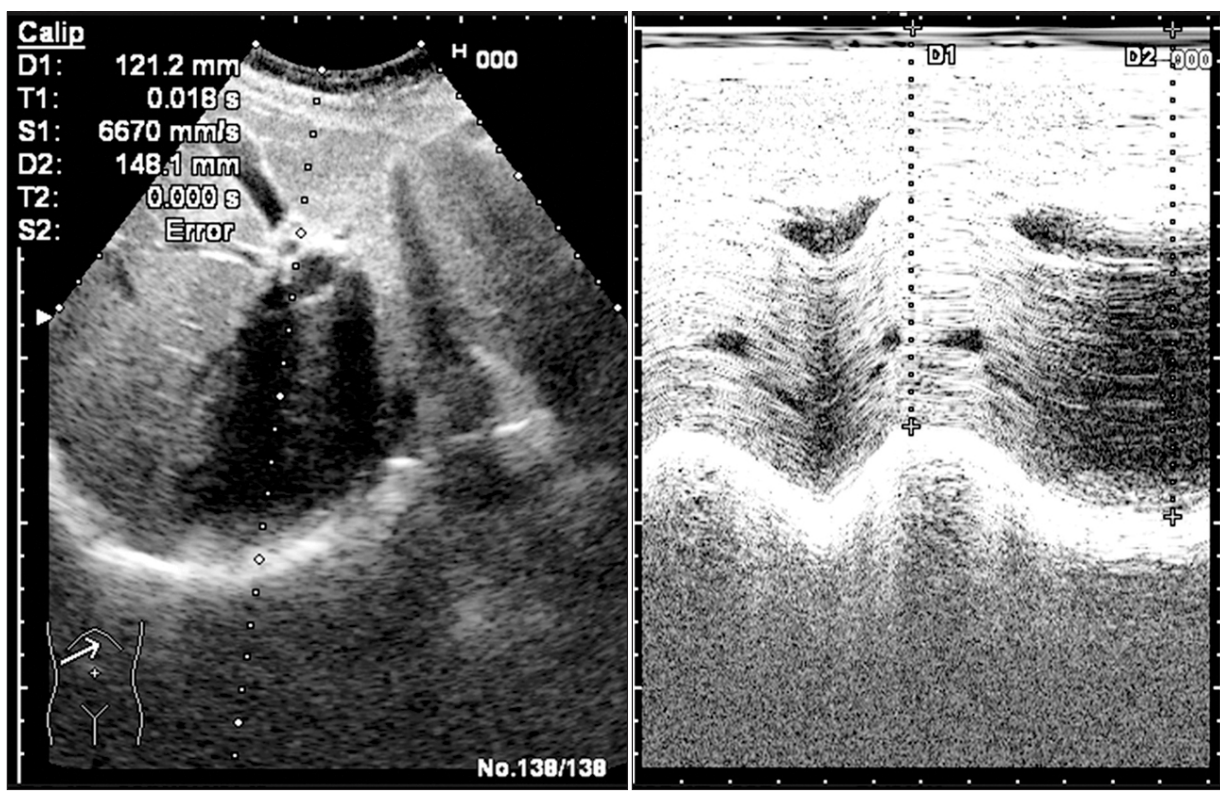

Figure 2. Minimal upward flexion of the tracing in M-mode signifying minimal diaphragmatic movement. 

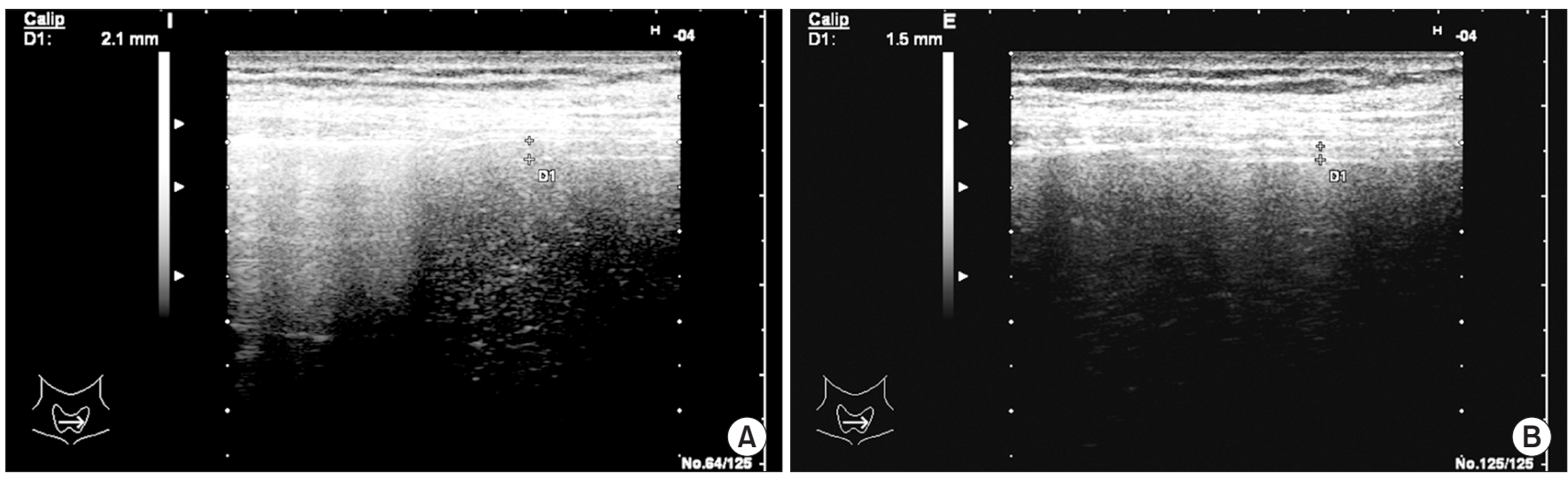

Figure 3. Diaphragmatic thickness assessment is shown. (A) The thickness during expiration. (B) The thickness during inspiration.

(increase in $\mathrm{RR}>30$ breaths per minute, heart rate $>120$ beats per minute, systolic blood pressure $<90 \mathrm{~mm} \mathrm{Hg}$ or $>180 \mathrm{~mm}$ $\mathrm{Hg}$, presence of confusion, agitation, diaphoresis, cyanosis, or evidence of increasing respiratory effort). The patients were followed until discharge or death.

A successful extubation was defined as spontaneous breathing for $>48$ hours following extubation. A failed extubation was defined as someone who was reintubated within 48 hours.

The patients were divided into two groups according to their extubation outcome: successfully extubated group and failed extubation group. The two groups were compared according to their age, laboratory results, lung mechanics, duration of mechanical ventilation, APACHE II score, and diaphragmatic function parameters.

\section{Statistical analysis}

Data was coded and entered by using the statistical package SPSS version 21 (IBM Corp., Armonk, NY, USA). The data were summarized by using number and percentage for qualitative variables. The quantitative data that were normally distributed were TV, Insp DT, and Exp DT. These data were presented by mean and standard deviation. Comparisons between the successful extubation and failed extubation groups regarding these normally distributed data were performed by using the independent sample t test.

The quantitative data that were not normally distributed were age, APACHE score, duration of mechanical ventilation, RR, RSBI, P/F ratio (arterial pressure of oxygen divided by the fraction of inspired oxygen expressed as a decimal), Cdyn, Cstat, DE, and DTF\%. These data were presented by median and interquartile range. Comparisons between the successful extubation and failed extubation groups regarding these data that were not normally distributed were performed by using the non-parametrical Mann-Whitney test.

To determine the best cut-off for DE and DTF parameters to predict successful extubation, we calculated area under the
64 Subjects included for SBT

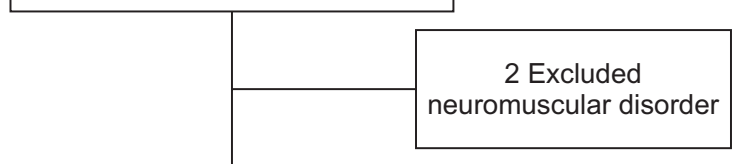

62 Successfully tolerated SBT

US was performed

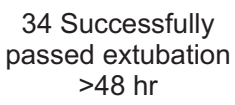

All subjects were extubated

Figure 4. A flow chart of patients included in the study. SBT: spontaneous brathing trial; US: ultrasonography.

receiving operating characteristic curve (ROC).

Correlation analysis was performed to test for linear relationships between variables. We accepted p-values $<0.05$ as indicative of statistical significance.

\section{Results}

The study included 64 patients who were ready for weaning; two patients showed signs of neuromuscular disorders and were excluded from the study. The remaining 62 patients were subjected to SBT and followed up for 48 hours to record extubation outcome (Figure 4). There were 26 (41.9\%) females and $36(58.1 \%)$ males. The included patients were diagnosed as follows: 15 (24.1\%) had chronic obstructive pulmonary disease, 14 (22.5\%) had overlap syndrome, 13 (20.9\%) 
had extensive pneumonia, six (9.6\%) had interstitial lung disease, four $(6.4 \%)$ had bronchiectasis, four $(6.4 \%)$ had sepsis, two (3.2\%) had lymphoma, two (3.2\%) had mediastinal mass, and two $(3.2 \%)$ were trauma patients.

As a cause of respiratory failure, 14 patients $(22.6 \%)$ presented with type 1 respiratory failure, and $48(77.4 \%)$ presented with type 2 respiratory failure.

History of ischemic heart diseases and cardiac problems were encountered in 16 patients $(25.8 \%)$, and a history of diabetes and metabolic syndrome was found in 15 patients (24.2\%).

Out of all patients, 28 (45\%) failed extubation and required reintubation and $34(55 \%)$ were successfully extubated and weaned.

The two groups did not significantly differ in age but differed in APACHE II score, which was significantly lower in the successful extubation group. As expected, the duration of mechanical ventilation was significantly higher in the failed extubation group versus the successful extubation group. As presented in Table 1, the parameters of lung mechanics represented by Cstat and Cdyn and the diaphragmatic function indices represented by Insp DT and Exp DT, DE, and DTF were significantly higher in the patients who succeeded in the trial of extubation (Figures 5, 6).

DE negatively correlated with the APACHE II score ( $\mathrm{r}=$ $-0.384, p=0.002$ ) as well as with the duration of mechanical ventilation $(\mathrm{r}=-0.494, \mathrm{p}<0.001)$ and positively correlated with dynamic compliance ( $\mathrm{r}=0.267, \mathrm{p}=0.036)$.

DTF negatively correlated with duration of mechanical ventilation $(\mathrm{r}=-0.373, \mathrm{p}=0.003)$ and positively correlated with dynamic compliance ( $\mathrm{r}=0.266, \mathrm{p}=0.037)$.

A ROC curve was used to assess the accuracy of DE and DTF in predicting weaning outcome. A cut-off value of DE of $>1.25 \mathrm{~cm}$ was associated with successful extubation, with a sensitivity of $97 \%$ and a specificity of $82 \%$. A DTF of $>21.5 \%$ predicts successful extubation with a sensitivity of $91.2 \%$ and a specificity of $60.7 \%$ (Figure 7).

\section{Discussion}

The process of weaning from mechanical ventilation is complex, with an estimated failure rate of $20 \%{ }^{14}$.

The diaphragm is considered to be the main muscle of respiration because it contributes approximately to $70 \%$ of the TV during inspiration in normal people ${ }^{15}$. Therefore, diaphragmatic dysfunction is one of the most important factors accounting for difficult weaning ${ }^{16}$. Ultrasound is a readily available, easy to use, and noninvasive imaging modality that is commonly used for procedures and clinical evaluation in the modern $\mathrm{ICU}^{17}$. Recently, ultrasound measurements have been used for evaluation of diaphragmatic contractility either by assessment of $\mathrm{DE}$ or $\mathrm{DT}^{17}$. In the current study, we showed that measurements of DE and DTF by ultrasound could be

Table 1. Differences in the blood gases, respiratory mechanics, and ultrasound parameters

\begin{tabular}{|c|c|c|c|}
\hline Variable & $\begin{array}{l}\text { Successful extubation } \\
\qquad(n=34)\end{array}$ & $\begin{array}{l}\text { Failed extubation } \\
\qquad(n=28)\end{array}$ & p-value \\
\hline Age, yr & $65(55-70)$ & $60.5(52.25-70)$ & $0.547^{*}$ \\
\hline Duration of MV, day & $4(3-5)$ & $8.5(6-10)$ & $<0.001^{* \dagger}$ \\
\hline $\mathrm{RR}$, breaths/min & $18.5(15-23.25)$ & $20(15-24)$ & $0.575^{*}$ \\
\hline $\mathrm{P} / \mathrm{F}$ ratio & $175(154-222.5)$ & $171(133.38-216.25)$ & $0.529^{*}$ \\
\hline Cdyn, $\mathrm{mL} / \mathrm{cm} \mathrm{H}_{2} \mathrm{O}$ & $19.9(14.25-26)$ & $13.7(11.45-18.03)$ & $0.004^{*}$ \\
\hline Cstat, $\mathrm{mL} / \mathrm{cm} \mathrm{H}_{2} \mathrm{O}$ & $35.65(19.78-47.7)$ & $23.3(16.18-32.2)$ & $0.020^{*}$ \\
\hline Insp DT, cm & $0.33 \pm 0.06$ & $0.25 \pm 0.07$ & $<0.001^{\dagger *}$ \\
\hline
\end{tabular}

Values are presented as median (IQR) or mean \pm SD.

*Mann-Whitney test. ${ }^{\dagger}$ Significant. ${ }^{*}$ Independent $t$ test.

APACHE II: Acute Physiology and Chronic Health Evaluation II; MV: mechanical ventilation; RR: respiratory rate; TV: tidal volume; RSBI: rapid shallow breathing index; P/F ratio: arterial pressure of oxygen divided by the fraction of inspired oxygen expressed as a decimal; Cdyn: dynamic compliance; Cstat: static compliance; Insp DT: inspiratory diaphragmatic thickness; Exp DT: expiratory diaphragmatic thickness; DE: diaphragmatic excursion; DTF\%: diaphragmatic thickness fraction; IQR: interquartile range; SD: standard deviation. 


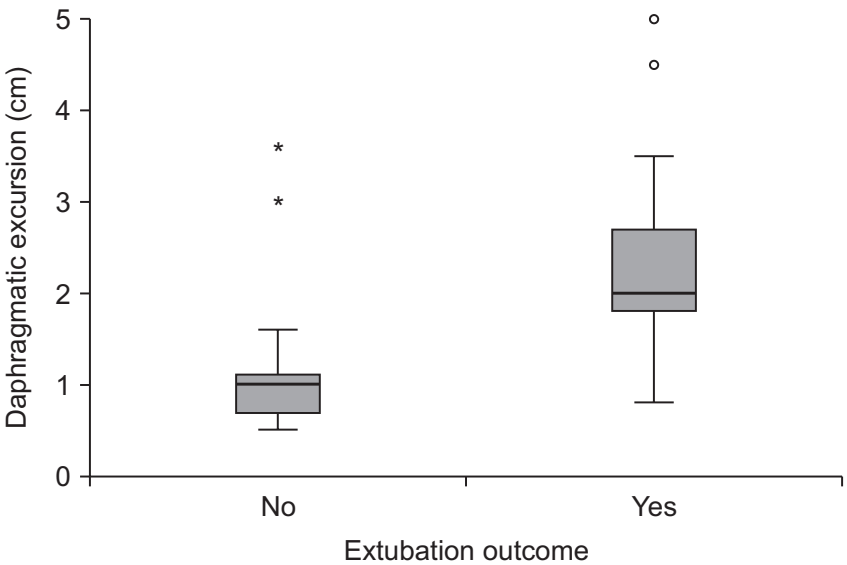

Figure 5. Difference in the diaphragmatic excursion between the weaned and non-weaned groups showing a high median of 2.00 with an interquartile range of 1.80-2.7 in the weaned group and of 1.00 with an interquartile range of 0.65-1.15 in the non-weaned group median.

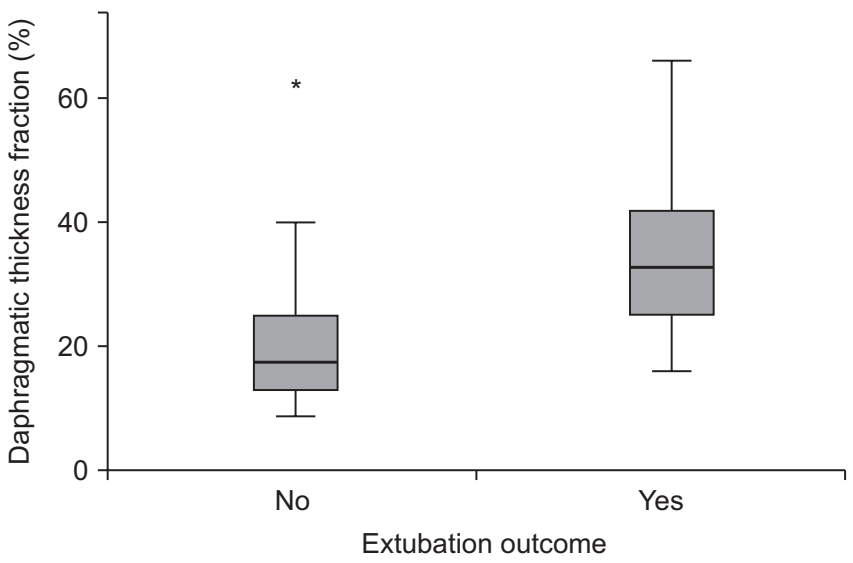

Figure 6. Difference in diaphragmatic thickness fraction between the weaned and non-weaned groups showing a median of $32.65 \%$ and an interquartile range of $24.50 \%$ to $34.25 \%$ in the weaned group and a median of $17.50 \%$ and an interquartile range of $13 \%$ to $25 \%$ in the non-weaned group.

used to predict successful weaning.

The patients were classified into successful extubation group and failed extubation group. There was no statistically significant difference in age between the groups, which meant that age was not a confounding factor that affected the extubation outcome, as would be expected, for example, in sarcopenia, which is age-related muscular loss.

Regarding the APACHE II score, there was a highly significant increase in the failed extubation group $(\mathrm{p}<0.001)$ (Table 1$)$ and a significant negative correlation between the APACHE II score and DE ( $\mathrm{p}=0.002)$ but not between the APACHE II score and DTF ( $p=0.053$ ). The APACHE II score contains some parameters that might affect the diaphragmatic function, such as

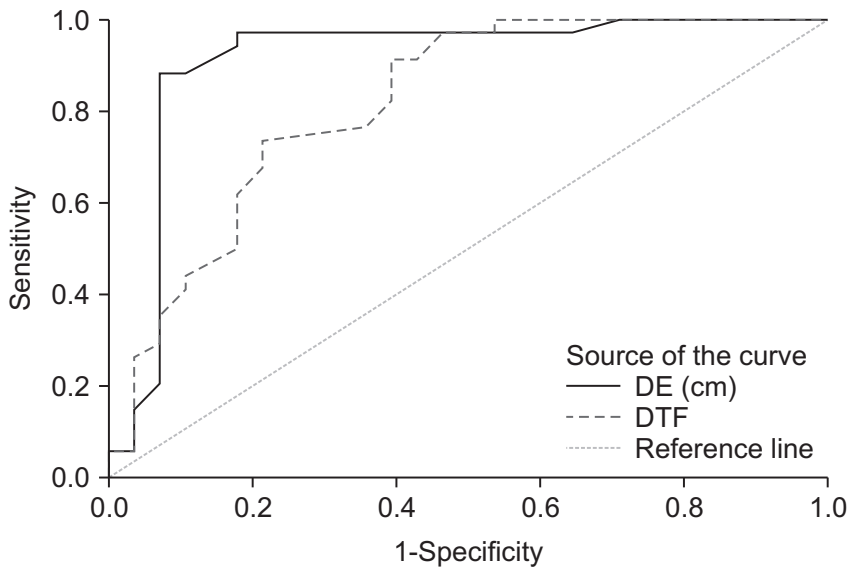

Figure 7. The receiver operating characteristic curve analysis of the sensitivity and specificity of both diaphragmatic excursion (DE) and diaphragmatic thickness fraction (DTF).

serum potassium, albumin, and hemoglobin levels. These results supported the conclusion that extubation failure was related to causes other than diaphragmatic factors. Additionally, the low significant negative correlation between the APACHE II score and DE and non-significant correlation between the APACHE II score and DTF parameters supported the conclusion that there were no large differences in the parameters affecting the diaphragm level between the two groups ${ }^{18}$. This finding could also be explained by the idea that DTF represents the number of fibers in the diaphragm, whereas DE represents the diaphragm function that can be affected by various measures included in the APACHE II score and explains why the score is correlated with the DE but not with the DTF.

The duration of mechanical ventilation was significantly different between the two groups (Table 1). Moreover, there were negative correlations between the duration of mechanical ventilation and DE and DTF. Ferrari et al. ${ }^{13}$ showed a statistically significant decrease in the duration of mechanical ventilation in the weaned group relative to that in the non-weaned group. Goligher et al. ${ }^{19}$ also discussed the same issue and concluded that changes in DT are common during mechanical ventilation and may be associated with diaphragmatic weakness. These results were in accordance with Grosu et al. ${ }^{20}$ where their study revealed that the duration of mechanical ventilation significantly predicted decreases in DT and strength. This finding can be explained by a previous findings that prolonged controlled mechanical ventilation in patients triggered significant reductions in the generation of both active and passive diaphragm myofibrillar force by reducing myofibrillar protein levels ${ }^{21}$ and that the duration of mechanical ventilation was correlated with DT, which also explained our finding above because a significant decrease in inspiratory DT at end inspiration by approximately $17 \%$ was observed on the third day of mechanical ventilation. This decrease continued progressively through the course of mechanical ventila- 
tion.

Regarding RSBI, there was no statistically significant difference between the two groups (Table 1) or when correlating RSBI with the DE and DTF. The RSBI is an integrative function of respiratory load and inspiratory muscle capacity that reflects the function of all inspiratory muscles, including the diaphragm, scalenes, para-sterna intercostals, and accessory inspiratory muscles (sternomastoids and external intercostals). If the diaphragm is failing (in a T-piece trial), the nondiaphragm inspiratory muscles will compensate to preserve $\mathrm{TV}$, and the presence of diaphragm weakness may be 'masked' by the increased contribution of the non-diaphragm inspiratory muscles ('rib cage' muscles) to TV. However, the rib cage muscles are more fatigable and weaker than the diaphragm and will not be able to sustain adequate ventilation. Accordingly, extubation failure may occur despite an initially acceptable TV and $\mathrm{RSBI}^{17}$. Consequently, RSBI can be considered to be a good parameter to start a weaning trial but not a predictor of weaning success, unlike the diaphragmatic function, which can predict the weaning outcome.

The diaphragm is an active respiratory muscle that contracts during inspiration, and the expiratory phase is passive, so it is main contribution should be at the inspiratory phase. The two groups differed significantly in the end inspiratory thickness of the diaphragm (Table 1). Comparing our study to that of Ferrari et al. ${ }^{13}$, who examined the DT as a weaning predictor, their study did not find a statistical difference in the DT during total lung capacity or residual volume between the two groups. We measured the DT during quiet breathing unlike in two previous studies ${ }^{12,13}$, which might explain the different levels of significance, although the numbers did not vary that much.

We found highly significant differences in DE and DTF between the two groups, findings that agree with those of most other recent studies (Table 1, Figure 7). Farghaly and Hasan ${ }^{16}$ found that the optimum cut-off of DE that predicted successful weaning was $\geq 10.5 \mathrm{~mm}$, with a sensitivity of $87.5 \%$ and a specificity of $71.5 \%$. Kim et al. ${ }^{7}$ determined that a DE $<10 \mathrm{~mm}$ measured during SBT was associated with weaning failure. Baess et al. $^{22}$ found that a DE $>1 \mathrm{~cm}$ was a predictor for weaning success. The slight difference in numerical values may be attributed to the different study populations.

DTF was significantly higher in the successful extubation group than in the failed extubation group (Figure 6). This result is consistent with the studies from Ferrari et al. ${ }^{13}$, DiNino et al. ${ }^{17}$, Dube et al. ${ }^{9}$, and Blumhof et al. ${ }^{3}$, which showed that their DTFs of $>36 \%,>30 \%,>29 \%$, and $>20 \%$, respectively, were consistent with weaning success. These studies also demonstrated the superiority of DTF versus RSBI in predicting weaning outcome, as mentioned above.

In a systematic review ${ }^{23}$ comparing different studies that assessed the contribution of DE and DTF in prediction of weaning outcome and compared the two parameters with each other, DTF was concluded to be superior over DE in prediction of weaning outcome, although in our study we found that DE showed superior sensitivity and specificity to those of DTF. Our finding can be explained by the timing in examining the diaphragm because our study measurements were made during a spontaneous breathing trial without the effect of positive-pressure ventilation. However, in the above-mentioned review, the data were obtained from measurements made during positive-pressure ventilation, which would affect the measured DE that is derived from adding the patient's effort to the pressure generated by the ventilator.

Diaphragmatic ultrasonography was found to be a reliable, noninvasive, and convenient technique for predicting weaning success. Incorporation of DTF and DE in the current weaning protocols will further enhance decision making regarding the optimum time for weaning and should improve weaning outcomes and avoid deleterious effects of prolonged intubations or premature extubations.

\section{Authors' Contributions}

Conceptualization: Elshazly MI, Assal HH. Methodology: Elkorashy RI, Kamel KM. Formal analysis: Ismail MS, Elshazly MI. Data curation: Ismail JH, Assal HH. Software: Ismail MS. Validation: Assal HH. Investigation: Ismail JH. Writing - original draft preparation: Ismail JH. Writing - review and editing: Assal HH. Approval of final manuscript: all authors.

\section{Conflicts of Interest}

No potential conflict of interest relevant to this article was reported.

\section{Funding}

No funding to declare.

\section{References}

1. Esteban A, Anzueto A, Frutos F, Alia I, Brochard L, Stewart TE, et al. Characteristics and outcomes in adult patients receiving mechanical ventilation: a 28-day international study. JAMA 2002;287:345-55.

2. Funk GC, Anders S, Breyer MK, Burghuber OC, Edelmann G, Heindl W, et al. Incidence and outcome of weaning from mechanical ventilation according to new categories. Eur Respir J 2010;35:88-94.

3. Blumhof S, Wheeler D, Thomas K, McCool FD, Mora J. Change in diaphragmatic thickness during the respiratory 
cycle predicts extubation success at various levels of pressure support ventilation. Lung 2016;194:519-25.

4. Blackwood B, Alderdice F, Burns K, Cardwell C, Lavery G, O'Halloran P. Use of weaning protocols for reducing duration of mechanical ventilation in critically ill adult patients: Cochrane systematic review and meta-analysis. BMJ 2011;342:c7237.

5. MacIntyre NR, Cook DJ, Ely EW Jr, Epstein SK, Fink JB, Heffner JE, et al. Evidence-based guidelines for weaning and discontinuing ventilatory support: a collective task force facilitated by the American College of Chest Physicians; the American Association for Respiratory Care; and the American College of Critical Care Medicine. Chest 2001;120(6 Suppl):375S-95S.

6. Nemer SN, Barbas CS, Caldeira JB, Carias TC, Santos RG, Almeida LC, et al. A new integrative weaning index of discontinuation from mechanical ventilation. Crit Care 2009;13:R152.

7. Kim WY, Suh HJ, Hong SB, Koh Y, Lim CM. Diaphragm dysfunction assessed by ultrasonography: influence on weaning from mechanical ventilation. Crit Care Med 2011;39:2627-30.

8. Nason LK, Walker CM, McNeeley MF, Burivong W, Fligner CL, Godwin JD. Imaging of the diaphragm: anatomy and function. Radiographics 2012;32:E51-70.

9. Dube BP, Dres M, Mayaux J, Demiri S, Similowski T, Demoule A. Ultrasound evaluation of diaphragm function in mechanically ventilated patients: comparison to phrenic stimulation and prognostic implications. Thorax 2017;72:811-8.

10. Kharma N. Dysfunction of the diaphragm: imaging as a diagnostic tool. Curr Opin Pulm Med 2013;19:394-8.

11. Laporta D, Grassino A. Assessment of transdiaphragmatic pressure in humans. J Appl Physiol (1985) 1985;58:1469-76.

12. Pirompanich P, Romsaiyut $S$. Use of diaphragm thickening fraction combined with rapid shallow breathing index for predicting success of weaning from mechanical ventilator in medical patients. J Intensive Care 2018;6:6.

13. Ferrari G, De Filippi G, Elia F, Panero F, Volpicelli G, Apra F.
Diaphragm ultrasound as a new index of discontinuation from mechanical ventilation. Crit Ultrasound J 2014;6:8.

14. Osman AM, Hashim RM. Diaphragmatic and lung ultrasound application as new predictive indices for the weaning process in ICU patients. Egypt J Radiol Nucl Med 2017;48:61-6.

15. Mead J. Functional significance of the area of apposition of diaphragm to rib cage. Am Rev Respir Dis 1979;119(2 Pt 2):31-2.

16. Farghaly S, Hasan AA. Diaphragm ultrasound as a new method to predict extubation outcome in mechanically ventilated patients. Aust Crit Care 2017;30:37-43.

17. DiNino E, Gartman EJ, Sethi JM, McCool FD. Diaphragm ultrasound as a predictor of successful extubation from mechanical ventilation. Thorax 2014;69:423-7.

18. Theerawit P, Eksombatchai D, Sutherasan Y, Suwatanapongched T, Kiatboonsri C, Kiatboonsri S. Diaphragmatic parameters by ultrasonography for predicting weaning outcomes. BMC Pulm Med 2018;18:175.

19. Goligher EC, Laghi F, Detsky ME, Farias P, Murray A, Brace D, et al. Measuring diaphragm thickness with ultrasound in mechanically ventilated patients: feasibility, reproducibility and validity. Intensive Care Med 2015;41:642-9.

20. Grosu HB, Lee YI, Lee J, Eden E, Eikermann M, Rose KM. Diaphragm muscle thinning in patients who are mechanically ventilated. Chest 2012;142:1455-60.

21. Farghaly S, Hasan AA, Makhlouf HA. Assessment of ventilator-induced diaphragmatic dysfunction in patients with chronic obstructive pulmonary disease using transthoracic ultrasonography. Egypt J Bronchol 2018;12:218-25.

22. Baess AI, Abdallah TH, Emara DM, Hassan M. Diaphragmatic ultrasound as a predictor of successful extubation from mechanical ventilation: thickness, displacement, or both? Egypt J Bronchol 2016;10:162-6.

23. Llamas-Alvarez AM, Tenza-Lozano EM, Latour-Perez J. Diaphragm and lung ultrasound to predict weaning outcome: systematic review and meta-analysis. Chest 2017;152:114050. 